\title{
MODELO DE CLASSIFICAÇÃO DE RISCO DE CRÉDITO DE EMPRESAS*
}

\author{
A MODEL FOR THE CLASSIFICATION OF COMPANIES’ CREDIT RISK
}

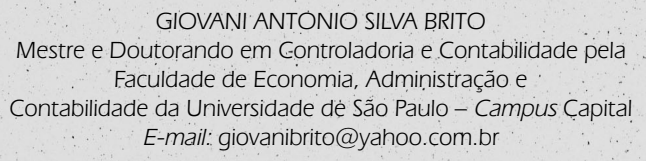

GIOVANI ANTONIO SILVA BRITO

Mestre e Doutorando em Controladoria e Contabilidade pela Faculdade de Economia, Administração e

Contabilidade da Universidade de Sắo Paulo - Campus Capital E-mail: giovanibrito@yahoo.com.br

\author{
ALEXANDRE ASSAF NETO \\ Professor Titular do Departamento de Contabilidade da \\ Faculdade de Economia, Administração e Contabilidade da \\ Universidade de São Paulo - Campus Ribeirão Preto \\ E-mail: assaf@terra.com.br
}

\section{RESUMO}

O processo de gerenciamento de risco de crédito em instituições financeiras vem passando por uma revisão ao longo dos últimos anos. Nesse contexto, diversas novas técnicas de mensuração de risco de crédito e tomadores têm sido desenvolvidas e implementadas por grandes Bancos. O objetivo desta pesquisa é desenvolver um modelo de classificação de risco para avaliar o risco de crédito de empresas no mercado brasileiro. O modelo foi construído com base em uma amostra de empresas de capital aberto classificadas como solventes ou insolventes no período entre 1994 e 2004. A técnica estatística utilizada no desenvolvimento do modelo foi a regressão logística. As variáveis independentes são índices financeiros calculados a partir das demonstrações contábeis e utilizados para representar a situação econômico-financeira das empresas. A validação do modelo foi efetuada utilizando o método Jackknife e uma Curva ROC. Os resultados do estudo indicam que o modelo de classificação de risco desenvolvido prevê eventos de default com um ano de antecedência com bom nível de acurácia. Os resultados, também, indicam que as demonstrações contábeis contêm informações que possibilitam a classificação das empresas como prováveis solventes ou prováveis insolventes.

Palavras-chave: Modelo de risco de crédito. Evento de default. Empresas de capital aberto. Regressão logística. Índices financeiros.

\section{ABSTRACT}

The process of credit risk management in financial institutions has been revised in recent years. In this context, large banks have developed and implemented several new techniques for measuring borrowers' credit risk. This research aims to develop a risk classification model to assess the credit risk of companies in the Brazilian market. The model was built based on a sample of publicly traded companies classified as solvent or insolvent during the period from 1994 to 2004. Logistic regression was used to develop the model. The independent variables of the model are financial ratios, calculated from the financial statements and used as proxies of companies' economic and financial situation. The validation of the model was done using the Jackknife method and a ROC Curve. The results of the study indicate that the risk classification model developed predicts default events one year prior to failure with good level of accuracy. The results also indicate that financial statements contain information that allow for the classification of companies as probably solvent or probably insolvent.

Keywords: Credit risk model: Default event. Publicly traded companies. Logistic regression. Financial ratios. 


\section{INTRODUÇÃO}

O processo de avaliação e gerenciamento de risco de crédito em instituições financeiras vem passando por um movimento de revisão ao longo dos últimos anos. Os métodos tradicionais de decisão baseados exclusivamente em critérios julgamentais têm perdido espaço nas atividades de crédito dos Bancos, que buscam instrumentos mais eficazes para mensurar o risco dos tomadores e das carteiras de crédito.

Nesse contexto, observa-se uma maior ênfase das instituições na utilização de modelos quantitativos como suporte às decisões de concessão de crédito e à gestão das carteiras. Os modelos de risco de crédito compõem um ferramental técnico que supre de informações os gestores e contribuem para que tomem decisões que atendam às diretrizes estabelecidas nas políticas de crédito da instituição.

O objetivo desta pesquisa é desenvolver um modelo de classificação de risco de crédito de grandes empresas que atuam no Brasil, utilizando a técnica estatística da regressão logística. O escopo do modelo é prever a ocorrência de eventos de default com a empresa no horizonte de tempo de um ano, visando, principalmente, a subsidiar os gestores no processo de concessão de crédito e gerenciamento de risco.

O modelo proposto estabelece uma relação estatística entre o default da empresa e um conjunto de índices econômico-financeiros calculados a partir das demonstrações contábeis. Com base nessa relação, é avaliado se as demonstrações contábeis fornecem informações que permitam aos seus diversos usuários prever a ocorrência de uma insolvência empresarial.

O restante deste artigo está organizado da seguinte maneira: na seção 2 são abordados conceitos sobre risco de crédito, na seção 3 são descritos os principais tipos de modelos de risco de crédito, na seção 4 é apresentado o modelo de classificação de risco desenvolvido e, por fim, na seção 5 são exibidas as conclusões e considerações finais do estudo.

\section{RISCO DE CRÉDITO}

O conceito de crédito pode ser analisado sob diversas perspectivas. Para uma instituição financeira, crédito refere-se, principalmente, à atividade de colocar um valor à disposição de um tomador de recursos sob a forma de um empréstimo ou financiamento, mediante compromisso de pagamento em uma data futura.

O crédito geralmente envolve a expectativa do recebimento de um valor em um certo período de tempo. Nesse sentido, Caouette et al. (1999, p.1) afirmam que o risco de crédito é a chance de que essa expectativa não se cumpra. De forma mais específica, o risco de crédito pode ser entendido como a possibilidade de o credor incorrer em perdas, em razão de as obrigações assumidas pelo tomador não serem liquidadas nas condições pactuadas.

Segundo Bessis (1998, p.81), o risco de crédito pode ser definido pelas perdas geradas por um evento de default do tomador ou pela deterioração da sua qualidade de crédito. Há diversas situações que podem caracterizar um evento de default de um tomador. O autor cita como exemplo o atraso no pagamento de uma obrigação, o descumprimento de uma cláusula contratual restritiva (covenant), o início de um procedimento legal como a concordata e a falência ou, ainda, a inadimplência de natureza econômica, que ocorre quando o valor econômico dos ativos da empresa se reduz a um nível inferior ao das suas dívidas, indicando que os fluxos de caixa esperados não são suficientes para liquidar as obrigações assumidas.

A deterioração da qualidade de crédito do tomador não resulta em uma perda imediata para a instituição financeira, mas sim no incremento da probabilidade de que um evento de default venha a ocorrer. Nos sistemas de classi- ficação de risco, as alterações na qualidade de crédito dos tomadores dão origem às chamadas migrações de risco. Cada instituição financeira adota seu próprio conceito de evento de default, que está normalmente relacionado ao atraso no pagamento de um compromisso assumido pelo tomador por períodos como 60 ou 90 dias.

O risco de crédito pode ser avaliado a partir dos seus componentes, que compreendem o risco de default, o risco de exposição e o risco de recuperação. O risco de default está associado à probabilidade de ocorrer um evento de default com o tomador em um certo período de tempo, o risco de exposição decorre da incerteza em relação ao valor do crédito no momento do default, enquanto o risco de recuperação se refere à incerteza quanto ao valor que pode ser recuperado pelo credor no caso de um default do tomador.

O risco de recuperação depende do tipo do default ocorrido e das características da operação de crédito, como valor, prazo e garantias. O risco de default é também tratado por "risco cliente", pois está vinculado às características intrínsecas do tomador de crédito. Os riscos de exposição e de recuperação são tratados por "risco operação", uma vez que estão associados a fatores específicos da operação de crédito.

A mensuração de risco de crédito é o processo de quantificar a possibilidade de a instituição financeira incorrer em perdas, caso os fluxos de caixa esperados com as operações de crédito não se confirmem. O risco de default constitui a principal variável desse processo, podendo ser definido como a incerteza em relação à capacidade de o devedor honrar os seus compromissos assumidos. 


\section{MODELOS DE RISCO DE CRÉDITO}

Os modelos de risco de crédito compreendem ferramentas e aplicações que têm por objetivo principal mensurar o risco de tomadores e transações individuais ou de uma carteira de crédito como um todo. Segundo Andrade (2005, p.1), os modelos de risco de crédito podem ser classificados em três grupos: modelos de classificação de risco, modelos estocásticos de risco de crédito e modelos de risco de portfolio.

Os modelos de classificação de risco buscam avaliar o risco de um tomador ou operação, atribuindo uma medida que representa a expectativa de risco de default, geralmente expressa na forma de uma classificação de risco (rating) ou pontuação (escore). Os modelos de classificação de risco são utilizados pelas instituições financeiras em seus processos de concessão de crédito.

Os modelos estocásticos de risco de crédito são aqueles que têm por objetivo avaliar o comportamento estocástico do risco de crédito ou das variáveis que o determinam. Esses modelos são utilizados pelas instituições

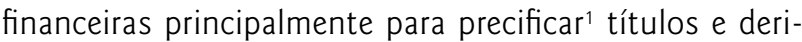
vativos de crédito.

Os modelos de risco de portfolio visam a estimar a distribuição estatística das perdas ou do valor de uma carteira de crédito, a partir da qual são extraídas medidas que quantificam o risco do portfolio. Esses modelos constituem uma importante ferramenta no processo de gestão de riscos das instituições, pois permitem que o risco de crédito seja avaliado de forma agregada, considerando os efeitos da diversificação produzidos pelas correlações entre os ativos da carteira. Os modelos de risco de portfolio, também, são utilizados para cálculo do capital econômico a ser alocado pela instituição.

Entre os modelos de classificação de risco, têm sido objeto de especial atenção por parte de pesquisadores os chamados modelos de previsão de insolvência. Os modelos de previsão de insolvência são aqueles que têm por objetivo principal medir a probabilidade de uma empresa incorrer em um evento de default ao longo de um certo período de tempo. Esses modelos são construídos a partir de uma amostra de casos históricos de empresas tomadoras de crédito, divididas em dois grupos: um que engloba as que incorreram em eventos de default, chamadas de insolven- tes, e outro que compreende as que não incorreram em default, chamadas de solventes.

A partir das características das empresas da amostra, são identificadas as variáveis que melhor discriminam as empresas que se tornaram insolventes e as que permaneceram solventes no período analisado. O conjunto de variáveis selecionadas é, então, utilizado para classificar as empresas proponentes de novas operações de crédito como prováveis solventes ou prováveis insolventes.

Um tipo de variável tradicionalmente utilizada para discriminar empresas solventes e insolventes são índices econômico-financeiros calculados a partir das demonstrações contábeis. Índices são relações entre contas ou grupos de contas das demonstrações contábeis que têm por objetivo evidenciar determinados aspectos da situação econômica e financeira da empresa.

A utilização de índices econômico-financeiros como variáveis explicativas em modelos de risco de crédito fundamenta-se no conceito de que o evento de default, geralmente, não é um processo abrupto. A deterioração da situação econômico-financeira da empresa tende a ocorrer de forma gradual, levando, em última instância, à degradação completa da sua qualidade de crédito e ao default. Como os índices evidenciam a deterioração da situação econômico-financeira da empresa ao longo do tempo, eles podem ser utilizados para prever a ocorrência do default.

Os modelos de previsão de insolvência geralmente se baseiam em técnicas estatísticas de análise multivariada, como regressão linear, análise discriminante e regressão logística. Mais recentemente, outras técnicas têm sido utilizadas no desenvolvimento de modelos de risco de crédito, como redes neurais e algoritmos genéticos. Uma técnica, também empregada nos últimos anos com a finalidade de prever a insolvência empresarial, é a análise por envoltória de dados (CASA NOVA; ONUSIC, 2005).

Os modelos que buscam prever a insolvência de empresas têm sido estudados há várias décadas nos meios acadêmicos. Algumas pesquisas se tornaram referência na literatura, como, por exemplo, os trabalhos de Beaver (1966), Altman (1968) e Ohlson (1980) em âmbito internacional, e os de Kanitz (1976), Matias (1978) e Altman et al. (1979) no Brasil.

\section{ESTUDO EMPÍRICO}

\subsection{Amostra de Empresas}

O objetivo central deste estudo é desenvolver um modelo de classificação de risco de crédito de grandes empresas. Na construção de um modelo de risco de crédito, o primeiro elemento a ser definido é o tipo de evento de default que se deseja prever. O conceito de default adotado nesta pesquisa é o início de um procedimento legal de concordata ou falência. Assume-se que, quando uma empresa se torna concordatária ou falida, a instituição credora incorre em perdas com as operações de crédito concedidas. Esse conceito de default foi utilizado em razão de ele atender aos propósitos do estudo, 
bem como de se tratar de uma informação disponível publicamente.

O modelo de risco de crédito foi desenvolvido a partir de uma amostra composta por empresas categorizadas como solventes e insolventes. O termo insolvente é utilizado para fazer referência a uma empresa que iniciou um processo de concordata ou falência durante o período analisado. Para os objetivos desta pesquisa, não há diferença entre a concordata e a falência, ambas caracterizando uma situação de insolvência.

A população de estudo, a partir da qual a amostra foi selecionada, compreende as empresas de capital aberto não financeiras, com papéis listados na Bolsa de Valores do Estado de São Paulo (Bovespa). Para a seleção da amostra, o primeiro procedimento adotado foi a identificação das empresas insolventes. A partir dos relatórios Boletim Diário de Informações - BDI e Suplemento de Orientação, publicados pela Bovespa, foram identificadas as empresas que tiveram ações negociadas como concordatárias no período compreendido entre os anos de 1994 e 2004. Além das concordatárias, também foram classificadas como insolventes as empresas que constaram do cadastro de companhias abertas da Comissão de Valores Mobiliários (CVM) na situação de falidas durante o mesmo período. Definiu-se como ano de ocorrência do evento de default, ou simplesmente ano do evento, aquele em que a empresa passou a ter suas ações negociadas como concordatária ou que passou a constar do cadastro da CVM como falida.

A partir da identificação das empresas insolventes, procedeu-se à seleção das empresas solventes que completaram a amostra. O grupo de solventes foi constituído selecionando-se, para cada empresa insolvente incluída na amostra, uma empresa solvente do mesmo setor econômico e de tamanho equivalente, segundo o valor dos seus ativos. A classificação setorial adotada foi a utilizada pela Economática.

A amostra final utilizada no desenvolvimento do modelo compreende 60 empresas, sendo 30 insolventes que se tornaram concordatárias ou falidas entre 1994 e 2004 e 30 solventes que foram emparelhadas com as primeiras. Esse método de emparelhamento da amostra foi baseado nos trabalhos de Beaver (1966), Altman (1968) e Sanvicente e Minardi (1998), podendo ser considerado como uma amostragem por julgamento.

Apesar de a quantidade de empresas da amostra não ser elevada, dois aspectos importantes devem ser citados. Em primeiro lugar, o número de defaults ocorridos com grandes empresas geralmente é reduzido. Entretanto, as perdas que podem ser incorridas pela instituição credora são significativas, uma vez que as operações com esse tipo de tomador geralmente têm valores elevados. Assim, o default de grandes empresas é tradicionalmente considerado como de baixa probabilidade e de elevado impacto.

Além desse aspecto, a população de estudo da qual a amostra foi selecionada engloba as empresas de capital aberto. Segundo dados obtidos junto à Bovespa, a quantidade média anual de companhias abertas entre 1994 e
2004 é de aproximadamente 250 empresas. Assim, podese considerar que o tamanho da amostra é proporcional à população de estudo e é coerente com o tipo de tomador considerado da pesquisa.

O Quadro 10 relaciona as empresas que compõem a amostra, seus setores de atuação e o ano do evento de default.

\subsection{Variáveis Explicativas}

As variáveis explicativas do modelo de classificação de risco de crédito compreendem índices utilizados para caracterizar a situação econômico-financeira das empresas, calculados a partir das suas demonstrações contábeis publicadas. O modelo proposto nesta pesquisa está fundamentado na relação entre a situação econômico-financeira da empresa e a ocorrência do evento de default. Dessa forma, quanto pior for a situação econômico-financeira de uma empresa, maior será a probabilidade de ela incorrer em default.

As demonstrações contábeis das empresas foram coletadas junto às bases de dados da Economática e da Comissão de Valores Mobiliários. Quando disponíveis, foram utilizadas as demonstrações consolidadas. Para as empresas insolventes, foram obtidas as três últimas demonstrações contábeis publicadas anteriormente ao ano do evento e, para as empresas solventes, foram coletadas as demonstrações referentes aos mesmos exercícios utilizados nas empresas insolventes com as quais foram emparelhadas.

Há um grande número de índices econômico-financeiros que potencialmente prevêem a ocorrência de um default. Em razão de inexistir uma teoria consolidada sobre esse assunto, foram testados 25 índices freqüentemente utilizados em estudos dessa natureza. Não foi objeto do trabalho a identificação de novos indicadores com poder de previsão de insolvência.

Os Quadros 20 e $3 \bullet$ apresentam, respectivamente, os 25 índices econômico-financeiros e a notação utilizada em suas fórmulas de cálculo.

Os índices econômico-financeiros utilizados no estudo compreendem indicadores de liquidez, de rentabilidade, de atividade, de estrutura, de análise dinâmica e de fluxo de caixa. A seguir, apresenta-se uma breve descrição desses indicadores. Informações detalhadas sobre índices econômico-financeiros podem ser obtidas em Assaf Neto (2002) e Silva (2003).

Os índices X1, X2, X3 e X4 são indicadores de liquidez que medem capacidade de pagamento, comparando direitos realizáveis e exigibilidades. Os índices X5, X6, X7 e X9 são indicadores de rentabilidade que avaliam os resultados gerados pela empresa. O índice X8 é um indicador de atividade que expressa a relação entre as vendas e os investimentos. O índice X10 é um indicador de cobertura de juros que mede a capacidade de a empresa pagar esses encargos. $\mathrm{O}$ índice X11 mede a parcela dos ativos que é financiada por recursos próprios. $\mathrm{O}$ índice $\mathrm{X} 12$ indica o volume de lucros retidos pela empresa em relação ao ativo. $O$ índice X13 mede a proporção de recursos próprios em relação aos recursos de terceiros na estrutura de capital. 


\begin{tabular}{|c|c|c|c|c|c|}
\hline Nr. & $\begin{array}{c}\text { Empresa } \\
\text { Insolvente }\end{array}$ & $\begin{array}{c}\text { Setor } \\
\text { Econômico }\end{array}$ & $\begin{array}{l}\text { Ano do } \\
\text { Evento }\end{array}$ & Nr. & $\begin{array}{l}\text { Empresa } \\
\text { Solvente }\end{array}$ \\
\hline 1 & Sibra & Siderurgia & 1994 & 31 & Belgo Mineira \\
\hline 2 & Ferro Ligas & Siderurgia & 1994 & 32 & Ferbasa \\
\hline 3 & Propasa & Papel e Celulose & 1995 & 33 & Votorantim \\
\hline 4 & Londrimalhas & Têxtil & 1995 & 34 & Karsten \\
\hline 5 & Aquatec & Química & 1995 & 35 & Bombril \\
\hline 6 & Mesbla & Comércio & 1995 & 36 & Lojas Renner \\
\hline 7 & Montreal & Participações & 1995 & 37 & Trevisa \\
\hline 8 & Inbrac & Eletroeletrônicos & 1995 & 38 & Trafo \\
\hline 9 & Cetenco & Construção & 1995 & 39 & Sultepa \\
\hline 10 & Cibran & Química & 1996 & 40 & Biobrás \\
\hline 11 & Velonorte & Têxtil & 1996 & 41 & Schlosser \\
\hline 12 & Iderol & Transporte & 1996 & 42 & Recrusul \\
\hline 13 & Trufana & Têxtil & 1996 & 43 & Têxtil Renaux \\
\hline 14 & Casa José Silva & Comércio & 1997 & 44 & Globex \\
\hline 15 & Pará de Minas & Têxtil & 1997 & 45 & Pettenati \\
\hline 16 & Corbetta & Curtume & 1997 & 46 & Vulcabrás \\
\hline 17 & Glasslite & Brinquedos & 1997 & 47 & Estrela \\
\hline 18 & SPSCS & Transporte & 1997 & 48 & Randon \\
\hline 19 & Tectoy & Eletroeletrônicos & 1998 & 49 & Gradiente \\
\hline 20 & Arapuã & Comércio & 1998 & 50 & Bompreço \\
\hline 21 & Copas & Química & 1999 & 51 & Fertibrás \\
\hline 22 & Adubos Trevo & Química & 1999 & 52 & Fosfertil \\
\hline 23 & Itaunense & Siderurgia & 1999 & 53 & Gerdau \\
\hline 24 & Lisamar & Alimentos & 2000 & 54 & Sadia \\
\hline 25 & Lorenz & Alimentos & 2000 & 55 & Granóleo \\
\hline 26 & Sharp & Eletroeletrônicos & 2000 & 56 & Electrolux \\
\hline 27 & Sano & Construção & 2002 & 57 & Sondotécnica \\
\hline 28 & Eucatex & Construção & 2003 & 58 & Duratex \\
\hline 29 & Chapecó & Alimentos & 2004 & 59 & Avipal \\
\hline 30 & Parmalat & Alimentos & 2004 & 60 & Leco \\
\hline
\end{tabular}

Quadro 1 Amostra de Empresas

Os índices X14, X15 e X16 são indicadores de estrutura que avaliam o grau de endividamento da empresa. $O$ índice X17 mede a parcela dos recursos próprios que está comprometida com $\mathrm{o}$ ativo permanente. $\mathrm{O}$ índice $\mathrm{X} 18$ indica $\mathrm{O}$ volume de estoques da empresa em relação ao seu ativo total. Os índices X19, X20, X21 e X22 são indicadores de análise dinâmica que avaliam a situação financeira da empresa. Os índices X23, X24 e X25 são indicadores de fluxo de caixa que medem os recursos gerados pelas atividades operacionais.

Os índices foram calculados com base nas demonstrações contábeis do penúltimo exercício anterior ao ano do evento de default. Não foram considerados os dados do último exercício, uma vez que a concordata de algumas empresas foi deferida antes que as demonstrações tivessem sido publicadas. Nesse caso, os índices do último exercício já poderiam estar refletindo a situação concordatária da empresa, o que prejudicaria a qualidade do modelo. Em relação a esse aspecto, Ohlson (1980, p.110) comenta que se o propósito do estudo é a previsão, utilizar as demonstrações do último exercício não é um procedimento adequado. A utilização dos dados do penúltimo exercício no modelo assegura um prazo de antecedência de pelo menos um ano em relação ao evento de default.

As estatísticas descritivas dos índices econômico-financeiros calculados revelam que alguns indicadores apresentam médias entre as empresas solventes e insolventes bastante próximas. Para avaliar se as diferenças das médias são significantes estatisticamente, foi aplicado o teste de médias (teste-t). O teste de igualdade de médias para duas amostras tem por objetivo estimar se as médias de duas populações são iguais estatisticamente. Se as médias dos índices de solventes e insolventes forem iguais estatisticamente, o indicador não é relevante para o modelo de risco de crédito.

O resultado do teste indica que, ao nível de significância de $5 \%$, não pode ser rejeitada a hipótese nula de igualdade de médias para os índices X6, X8, X9, X18, X20, X23, X24 e X25. Os valores médios desses indicadores para os 


\begin{tabular}{|c|c|c|}
\hline Código & Índice & Fórmula \\
\hline $\mathrm{X} 1$ & Liquidez geral & $(A C+R L P) /(P C+E L P)$ \\
\hline $\mathrm{X} 2$ & Liquidez corrente & $A C / P C$ \\
\hline X3 & Liquidez seca & (AC - ESTOQUES) / PC \\
\hline $\mathrm{X} 4$ & Liquidez imediata & DISPONÍVEL / PC \\
\hline$\times 5$ & Retorno sobre o patrimônio líquido & LL / PL inicial \\
\hline X6 & Retorno sobre o ativo & LAJIR / AT \\
\hline$x 7$ & Retorno sobre vendas & $\mathrm{LL} / \mathrm{VL}$ \\
\hline $\mathrm{x} 8$ & Giro do ativo & $\mathrm{VL} / \mathrm{AT}$ \\
\hline X9 & Margem operacional & LAJIR / VL \\
\hline $\mathrm{X} 10$ & Lucro operacional sobre despesas financeiras & LAJIR / DF \\
\hline $\mathrm{x} 11$ & Patrimônio líquido sobre ativo & $\mathrm{PL} / \mathrm{AT}$ \\
\hline $\mathrm{X} 12$ & Lucros retidos sobre ativo & $(L A+R L) / A T$ \\
\hline $\mathrm{X} 13$ & Patrimônio líquido sobre exigível total & $\mathrm{PL} /(\mathrm{PC}+\mathrm{ELP})$ \\
\hline $\mathrm{X} 14$ & Endividamento total & $(P C+E L P) / A T$ \\
\hline$\times 15$ & Endividamento de curto prazo & PC / AT \\
\hline $\mathrm{X} 16$ & Endividamento financeiro & $(P C F+E L P F) / A T$ \\
\hline $\mathrm{X} 17$ & Imobilização do patrimônio líquido & $\mathrm{AP} / \mathrm{PL}$ \\
\hline $\mathrm{X} 18$ & Estoques sobre ativo & ESTOQUES / AT \\
\hline $\mathrm{X} 19$ & Capital de giro líquido & $(A C-P C) / A T$ \\
\hline $\mathrm{X} 20$ & Necessidade de capital de giro & $(A C O-P C O) / A T$ \\
\hline $\mathrm{X} 21$ & Saldo de tesouraria sobre ativo & (ACF - PCF) / AT \\
\hline$\times 22$ & Saldo de tesouraria sobre vendas & $(\mathrm{ACF}-\mathrm{PCF}) / \mathrm{VL}$ \\
\hline $\mathrm{X} 23$ & Fluxo de caixa operacional sobre ativo & FCO / AT \\
\hline$\times 24$ & Fluxo de caixa operacional sobre exigível total & $\mathrm{FCO} /(\mathrm{PC}+\mathrm{ELP})$ \\
\hline X25 & Fluxo de caixa operacional sobre endiv. financeiro & $\mathrm{FCO} /(\mathrm{PCF}+\mathrm{ELPF})$ \\
\hline
\end{tabular}

Quadro 2 IÍndices Econômico-Financeiros

\begin{tabular}{|c|l|c|l|}
\hline \multicolumn{3}{|c|}{ Notação } \\
\hline AC & Ativo circulante & LAJIR & Lucro antes dos juros e imposto de renda \\
\hline ACF & Ativo circulante financeiro & $\mathrm{LL}$ & Lucro líquido \\
\hline ACO & Ativo circulante operacional & PC & Passivo circulante \\
\hline AP & Ativo permanente & PCF & Passivo circulante financeiro \\
\hline AT & Ativo total & PCO & Passivo circulante operacional \\
\hline DF & Despesas financeiras & PL & Patrimônio líquido \\
\hline ELP & Exigível a longo prazo & RL & Reserva de Lucros \\
\hline ELPF & Exigível a longo prazo financeiro & RLP & Realizável a longo prazo \\
\hline FCO & Fluxo de caixa das operações & VL & Vendas líquidas \\
\hline LA & Lucros Acumulados & & \multicolumn{2}{|l}{} \\
\hline
\end{tabular}

Quadro 3 |Notação das Fórmulas de Cálculo dos Índices

grupos de solventes e insolventes não apresentaram diferenças estatisticamente significantes, portanto, eles não foram utilizados no modelo de risco de crédito. Os demais índices econômico-financeiros apresentaram diferenças de médias com significância estatística. A Tabela 10 apresenta os resultados do teste.

\subsection{Técnica Estatística}

O modelo de risco de crédito foi desenvolvido utilizando-se a técnica estatística da regressão logística. A regressão logística, ou análise logit, é uma técnica de análise multivariada, apropriada para as situações nas quais a variável dependente é categórica e assume um entre dois resultados possíveis (binária), tais como: "normal ou anormal", "cliente ou não cliente" e "solvente ou insolvente".

O objetivo da regressão logística é gerar uma função matemática cuja resposta permita estabelecer a probabilidade de uma observação pertencer a um grupo previamente determinado, em razão do comportamento de um conjunto de variáveis independentes. Os coeficientes esti- 
Tabela 1 Teste de Igualdade de Médias para os Índices Econômico-Financeiros

\begin{tabular}{|c|c|c|}
\hline \multirow{2}{*}{ Índice } & \multicolumn{2}{|c|}{ Teste-t de Igualdade de Médias } \\
\hline & Estatística t & Valor $\mathbf{p}$ \\
\hline $\mathrm{X} 1$ & 3,495 & 0,001 \\
\hline$\times 2$ & 5,549 & 0,000 \\
\hline$\times 3$ & 4,933 & 0,000 \\
\hline $\mathrm{X} 4$ & 4,769 & 0,000 \\
\hline$\times 5$ & 2,886 & 0,005 \\
\hline $\mathrm{x} 6$ & 0,534 & 0,595 \\
\hline$\times 7$ & 2,551 & 0,013 \\
\hline $\mathrm{x} 8$ & $-0,149$ & 0,882 \\
\hline $\mathrm{x} 9$ & 0,253 & 0,801 \\
\hline$\times 10$ & 2,935 & 0,005 \\
\hline $\mathrm{X} 11$ & 3,900 & 0,000 \\
\hline $\mathrm{X} 12$ & 4,407 & 0,000 \\
\hline $\mathrm{X} 13$ & 4,211 & 0,000 \\
\hline $\mathrm{X} 14$ & $-3,662$ & 0,001 \\
\hline $\mathrm{X} 15$ & $-4,168$ & 0,000 \\
\hline $\mathrm{X} 16$ & $-6,642$ & 0,000 \\
\hline $\mathrm{X} 17$ & $-3,424$ & 0,001 \\
\hline$\times 18$ & 0,961 & 0,341 \\
\hline$\times 19$ & 5,673 & 0,000 \\
\hline$\times 20$ & 0,629 & 0,532 \\
\hline$\times 21$ & 8,347 & 0,000 \\
\hline$\times 22$ & 6,121 & 0,000 \\
\hline$x 23$ & 1,416 & 0,162 \\
\hline$\times 24$ & 0,431 & 0,668 \\
\hline$\times 25$ & 0,274 & 0,785 \\
\hline
\end{tabular}

mados pelo modelo de regressão indicam a importância de cada variável independente para a ocorrência do evento.

A regressão logística apresenta certas vantagens em relação à análise discriminante linear, principalmente devido às suas suposições iniciais serem menos rígidas. A análise discriminante linear está baseada em uma série de pressupostos bastante restritivos, como a normalidade das variáveis independentes e a igualdade das matrizes de variância-covariância dos grupos de interesse. Essas suposições podem não ser válidas em muitas situações práticas de análise de risco de crédito, principalmente quando há variáveis independentes de natureza não métrica. Segundo Hair et al. (1998, p.276), a regressão logística não assume esses rígidos pressupostos e é uma técnica bem mais robusta quando eles não são atendidos.

Outro aspecto que favorece a utilização da regressão logística é que seus resultados podem ser interpretados em termos de probabilidade. Esse fator se mostra particularmente importante nos modelos de risco de crédito, pois possibilita que seja medida a probabilidade de um determinado tomador assumir a condição de solvente ou insolvente, em face de um conjunto de atributos. Em relação à análise discriminante linear, Ohlson (1980, p.112) comenta que o resultado da equação discriminante é um escore que tem pouca interpretação intuitiva, pois ele é basicamente um dispositivo discriminatório de classificação ordinal.
Na regressão logística, há uma transformação na variável dependente, que é convertida em uma razão de probabilidades e posteriormente em uma variável de base logarítmica (transformação logística). Devido à natureza não linear dessa transformação, os coeficientes da regressão são estimados pelo método da máxima verossimilhança. $O$ modelo da regressão logística assume a seguinte relação:

$$
\operatorname{In}\left(\frac{p}{1-p}\right)=b_{0}+b_{1} X_{1}+b_{2} X_{2}+\ldots+b_{k} X_{k}
$$

em que $p$ é a probabilidade de ocorrer o evento; $1-p$ a probabilidade de não ocorrer o evento; $p /(1-p)$ a razão de probabilidades; $x_{1}$ as variáveis independentes e $b_{1}$ os coeficientes estimados.

$\mathrm{Na}$ regressão logística, os coeficientes medem o efeito de alterações nas variáveis independentes sobre o logaritmo natural da razão de probabilidades, chamado de logit. Para avaliar o impacto dos parâmetros sobre a probabilidade de ocorrer o evento, eles devem ser transformados por meio de antilogaritmo. A probabilidade associada à ocorrência do evento de interesse pode ser obtida pela expressão:

$$
p=\frac{1}{1+e^{-\left(b_{0}+b_{1} X_{1}+b_{2} X_{2}+\ldots+b_{k} X_{k}\right)}}
$$

em que e é base dos logaritmos naturais (aproximadamente 2,718$)$. 


\subsection{Desenvolvimento do Modelo}

Após a seleção da amostra de empresas, o cálculo dos índices econômico-financeiros e a definição da técnica de análise de dados, procedeu-se ao desenvolvimento do modelo. O objetivo desse procedimento é identificar uma relação matemática entre as variáveis explicativas e o estado de solvência ou insolvência das empresas, de forma que o conjunto de índices possa ser utilizado para estimar a probabilidade de futuros eventos de default.

No modelo logit, a variável dependente pode assumir um valor entre zero e um. Neste estudo, foi atribuído o valor zero para indicar estado de solvência e o valor um para estado de insolvência. O ponto de corte do modelo é 0,5 , portanto, as empresas com resultado inferior a 0,5 são classificadas como solventes e as empresas com resultado superior a esse valor são classificadas como insolventes. As variáveis independentes testadas foram os 17 índices cujas médias entre solventes e insolventes apresentaram diferenças estatisticamente significantes.
O método de seleção das variáveis foi o forward stepwise, pelo critério do menor Likelihood Ratio. O método stepwise é baseado em um algoritmo estatístico que avalia a importância de cada variável independente e as inclui ou exclui do modelo segundo uma determinada regra. A importância de cada variável é definida em termos de uma medida de significância estatística do seu coeficiente. Os parâmetros utilizados foram $5 \%$ de significância para a entrada das variáveis e $10 \%$ para a saída. Também foram testados o método do maior coeficiente Wald e o método da maior probabilidade condicional de máxima verossimiIhança, os quais produziram resultados idênticos.

O modelo final foi composto pelo intercepto e quatro variáveis explicativas. As variáveis incluídas no modelo foram os índices X12 (lucros retidos sobre ativo), X16 (endividamento financeiro), X19 (capital de giro líquido) e X22 (saldo de tesouraria sobre vendas). A função matemática do modelo é:

$$
\operatorname{In}\left(\frac{p}{1-p}\right)=-4,740-4,528 . X_{12}+18,433 \cdot X_{16}-14,080 \cdot X_{19}-11,028 . X_{22}
$$

Os coeficientes das variáveis apresentaram o sinal esperado. As variáveis X12, X19 e X22 possuem coeficientes negativos, indicando que quanto maiores os valores assumidos por esses índices, menor a probabilidade de a empresa sofrer um default. Por outro lado, a variável X16 apresentou coeficiente positivo, mostrando que quanto maior o valor assumido por esse indicador, maior a probabilidade de a empresa se tornar insolvente.

Um elemento importante a ser considerado nos modelos de regressão é a correlação entre as variáveis independentes. A inclusão de variáveis altamente correlacionadas não é desejável, pois essas variáveis, chamadas de colineares, fornecem informações similares para explicar o comportamento da variável dependente, prejudicando a capacidade preditiva do modelo. A Tabela $2 \boldsymbol{O}$ apresenta os coeficientes de correlação de Pearson das variáveis do modelo. Conforme se verifica, os índices selecionados não são altamente correlacionados.

Para avaliar a capacidade de previsão do modelo, pode-se construir uma matriz de classificação para mostrar a quantidade de empresas classificadas correta e incorretamente pelo modelo. Conforme demonstra a matriz na Tabela $3 \mathbf{0}$, o nível de acerto do modelo desenvolvido foi de $90 \%$, tendo sido classificadas corretamente 54 das 60 empresas da amostra.

Do grupo de solventes, 28 empresas foram classificadas corretamente e 2 classificadas erradamente, o que representa uma taxa de acerto de 93,3\%. Em relação ao grupo de insolventes, houve 26 classificações corretas e 4 erradas, o que corresponde a uma taxa de acerto de $86,7 \%$. O erro tipo I, classificar uma empresa insolvente como sol-

Tabela 2 || Matriz de Correlação das Variáveis Explicativas

\begin{tabular}{c|c|c|c|c} 
& $\mathbf{X 1 2}$ & $\mathbf{X 1 6}$ & $\mathbf{X 1 9}$ & $\mathbf{X 2 2}$ \\
$\mathbf{X 1 2}$ & 1 & & & \\
\hline $\mathbf{X 1 6}$ & $-0,39$ & 1 & & \\
\hline $\mathbf{X 1 9}$ & 0,29 & $-0,45$ & 1 & \\
\hline $\mathbf{X 2 2}$ & 0,54 & $-0,51$ & 0,47 & 1
\end{tabular}

Tabela 3 || Matriz de Classificação do Modelo de Risco de Crédito

\begin{tabular}{l|c|c|c|c}
\multirow{2}{*}{ Observado } & \multicolumn{2}{|c|}{ Estimado } & \multirow{2}{*}{ Total } & $\begin{array}{c}\text { Classificações } \\
\text { Corretas }\end{array}$ \\
\cline { 2 - 3 } Solventes & Solventes & Insolventes & & 30 \\
\hline Insolventes & 28 & 2 & $30,3 \%$ \\
\hline Total & 4 & 26 & 30 & $86,7 \%$ \\
\hline
\end{tabular}


vente, foi de $13,3 \%$, enquanto o erro tipo II, classificar uma empresa solvente como insolvente, foi de $6,7 \%$.

\subsection{Avaliação do Ajuste do Modelo}

Para avaliar a qualidade de um modelo logístico, diversos testes e medidas podem ser utilizados. O primeiro passo é avaliar a significância das variáveis explicativas incluídas no modelo, o que é feito por meio de um teste QuiQuadrado, sob a hipótese nula de que todos os coeficientes são iguais a zero. O resultado da aplicação desse teste no modelo desenvolvido teve estatística Qui-Quadrado de 60,112, com significância de 0,000, o que leva à rejeição da hipótese nula e à interpretação de que ao menos um dos coeficientes é estatisticamente diferente de zero.

A análise da significância estatística de cada coeficiente é feita com base no teste Wald. Semelhante ao teste $t$ aplicado aos modelos lineares, o teste Wald avalia a hipótese nula de que o parâmetro estimado é igual a zero. A estatística Wald tem distribuição Qui-Quadrado, sendo calculada pelo quadrado da razão entre o coeficiente e o seu erro padrão. Os resultados do teste Wald para o modelo proposto indicam que os parâmetros das quatro variáveis independentes selecionadas são estatisticamente diferentes de zero. A Tabela $4 \boldsymbol{O}$ apresenta as estimativas dos parâmetros das variáveis do modelo, bem como os erros padrão, as estatísticas Wald, os graus de liberdade e os valores de probabilidade do teste Wald.

A avaliação do nível de ajuste de um modelo logístico pode ser realizada por meio do Likelihood Value, dos pseudo $\mathrm{R}^{2}$ e do teste de Hosmer e Lemeshow (1980). O Likelihood Value é uma medida da qualidade geral do modelo e quanto menor o seu valor, melhor é o ajuste do modelo como um todo. O Cox-Snell R² e o Nagelkerke R² são medidas que se assemelham ao coeficiente de determinação da regressão linear. Maiores valores dessas medidas indicam melhor ajuste do modelo. O Cox-Snell R² baseia-se no Likelihood Value e tem uma escala que começa em zero mas não alcança um em seu limite superior. O Nagelkerke $\mathrm{R}^{2}$ é similar ao CoxSnell $\mathrm{R}^{2}$, porém tem uma escala que vai de zero a um.

A Tabela 50 apresenta os valores do Likelihood Value, Cox-Snell R2 e Nagelkerke R² do modelo. Os dados evidenciam a redução do Likelihood Value e o aumento do Cox-Snell R² e do Nagelkerke R2 proporcionado pela inclusão das variáveis explicativas em cada etapa do processo stepwise. As variáveis foram incluídas na seguinte seqüência: X16, X19, X22 e X12.

O teste de Hosmer e Lemeshow (1980) avalia as diferenças entre as classificações previstas pelo modelo e as observadas. Se as diferenças forem significativas, o grau de acurácia do modelo não é bom. O teste divide os casos em classes e compara as freqüências preditas e observadas em cada classe por meio de uma estatística Qui-Quadrado. A Tabela $6 \mathbf{O}$ apresenta os resultados finais do teste. Ao nível de significância de 5\%, aceita-se a hipótese nula de que não há diferenças significativas entre os valores preditos e observados, o que indica que o modelo é capaz de produzir estimativas e classificações confiáveis.

\subsection{Validação do Modelo}

O modelo desenvolvido classificou corretamente $90 \%$ das empresas da amostra. No entanto, ao se testar o modelo com a própria amostra utilizada para a estimação dos seus parâmetros, pode-se concluir que o seu desempenho

Tabela 4 \ Coeficientes do Modelo de Risco de Crédito

\begin{tabular}{c|c|c|c|c|c} 
Variável & Coeficiente & Erro-Padrão & Teste Wald & g.l. & Valor-p \\
Intercepto & $-4,740$ & 2,165 & 4,793 & 1 & 0,029 \\
\hline X12 & $-4,528$ & 2,414 & 3,519 & 1 & 0,061 \\
\hline$X 16$ & 18,433 & 8,297 & 4,936 & 1 & 0,026 \\
\hline$X 19$ & $-14,080$ & 6,998 & 4,048 & 1 & 0,044 \\
\hline X22 & $-11,028$ & 6,103 & 3,265 & 1 & 0,071
\end{tabular}

Tabela 5 || Likelihood Value, Cox-Snell $R^{2}$ e Nagelkerke $R^{2}$

\begin{tabular}{c|c|c|c} 
Step & Likelihood Value & Cox-Snell R & Nagelkerke $\mathbf{R}^{\mathbf{2}}$ \\
\hline 1 & 50,103 & 0,424 & 0,565 \\
\hline 2 & 36,454 & 0,541 & 0,721 \\
\hline 3 & 27,761 & 0,603 & 0,804 \\
\hline 4 & 23,066 & 0,633 & 0,844
\end{tabular}

Tabela 6 || Teste Hosmer e Lemeshow

\begin{tabular}{c|c|c|c} 
Step & Qui-Quadrado & g.I. & Valor-p \\
\hline 1 & 4,798 & 8 & 0,779 \\
\hline 2 & 5,892 & 8 & 0,659 \\
\hline 3 & 3,191 & 8 & 0,922 \\
\hline 4 & 2,022 & 8 & 0,980
\end{tabular}


é bom quando, na realidade, ele pode funcionar bem apenas para essas observações. Assim, para avaliar se o modelo mantém o seu poder preditivo para outras amostras provindas da mesma população, são necessários testes para a sua validação. Segundo Hosmer e Lemeshow (2000, p.186), a validação do modelo é especialmente importante quando ele é usado com a finalidade de previsão de resultados.

A validação do modelo foi feita por meio do método baseado em múltiplas sub-amostras denominado jackknife, proposto por Lachenbruch (1967). O método jackknife é uma técnica estatística amplamente aceita na validação de modelos de classificação e é particularmente útil para pesquisas que envolvam amostras de tamanhos pequenos, pois permite que todas as observações sejam utilizadas na estimação dos parâmetros do modelo (NEOPHYTOU et al., 2000, p.20).

O método está baseado no princípio "leave-one-out" e consiste em separar uma observação da amostra original, estimar os coeficientes do modelo com base no restante da amostra (n-1) e classificar a observação apartada utilizando a nova equação. O procedimento é repetido para toda a amostra (n vezes), de maneira que todas as observações sejam classificadas por modelos cujos parâmetros foram estimados com base nas demais. O percentual de classificações corretas é acumulado para todas as observações da amostra, indicando a precisão global do modelo. A Tabela $7 \boldsymbol{O}$ apresenta a matriz de classificação obtida com a aplicação do método jackknife. O percentual de acerto acumulado foi de $88,3 \%$, tendo sido classificadas incorretamente sete empresas da amostra $(11,7 \%)$, três do grupo de solventes e quatro do grupo de insolventes.
Um segundo procedimento utilizado para avaliar a performance do modelo foi a construção de uma Curva ROC, conforme sugerido por Oliveira e Andrade (2002, p.3). A curva ROC (Receiver Operating Characteristic) constitui uma técnica bastante útil para validar modelos de risco de crédito e está baseada nos conceitos da sensitividade e da especificidade. A sensitividade é a proporção de acerto na previsão da ocorrência de um evento nos casos em que ele de fato ocorreu. A especificidade é proporção de acerto na previsão da não ocorrência de um evento nos casos em que ele de fato não ocorreu.

Para a construção da Curva ROC, são calculadas a sensitividade e a especificidade para todas as observações da amostra, considerando diferentes pontos de corte do modelo. A curva é obtida registrando em um gráfico "sensitividade" $x$ "1 - especificidade" para os diversos pontos de corte. A área sob a curva mede a capacidade de discriminação do modelo. Hosmer e Lemeshow (2000, p.162) apresentam uma regra geral para avaliação do resultado da área sob a Curva ROC:

a) área no intervalo entre 0,7 e 0,8: discriminação aceitável;

b) área no intervalo entre 0,8 e 0,9: excelente discriminação;

c) área acima de 0,9: excepcional discriminação.

A Curva ROC do modelo de risco de crédito, representada no Gráfico $1 \boldsymbol{\nabla}$, revela que a área sob a curva é de 0,978. Segundo a escala proposta pelos autores citados, esse valor indica um excepcional poder de discriminação do modelo.

Tabela 7 | Matriz de Classificação - Validação do Modelo

\begin{tabular}{l|c|c|c|c}
\multirow{2}{*}{ Observado } & \multicolumn{2}{|c|}{ Estimado } & \multirow{2}{*}{ Total } & $\begin{array}{c}\text { Classificações } \\
\text { Corretas }\end{array}$ \\
\cline { 2 - 3 } Solventes & Solventes & Insolventes & & 30 \\
\hline Insolventes & 27 & 03 & 30 & $90,0 \%$ \\
\hline Total & 31 & 26 & 60 & $86,7 \%$ \\
\hline
\end{tabular}

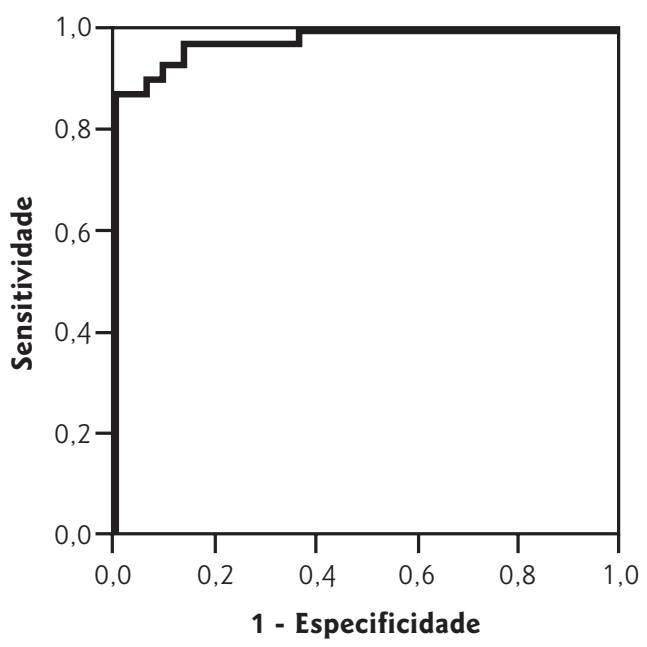

Gráfico 1 - Curva ROC 


\section{CONSIDERAÇÕES FINAIS}

O objetivo deste estudo foi desenvolver um modelo de classificação de risco de crédito de grandes empresas que atuam no Brasil. Utilizando a técnica estatística da regressão logística e um conjunto de quatro índices econômicofinanceiros como variáveis explicativas, o modelo proposto possibilita a previsão da ocorrência de eventos de default com um ano de antecedência, alcançando significativo índice de acerto.

O modelo de risco de crédito desenvolvido estabelece uma relação entre o evento de default e a situação econômico-financeira da empresa, caracterizada por meio de índices calculados a partir das suas demonstrações contábeis. Com base nessa relação, foi possível testar o conteúdo informacional das demonstrações contábeis das empresas de capital aberto publicadas no Brasil. Concluiu-se que as demonstrações contábeis fornecem informações que permitem classificar as empresas como prováveis solventes ou prováveis insolventes, com bom nível de precisão.

Por fim, destaque-se que os modelos de risco de crédito derivados empiricamente, a exemplo dos modelos de classificação de risco, têm sido objeto de críticas nos meios acadêmicos, em razão de eles não estarem suportados por uma teoria explícita. No entanto, embora esses modelos não tenham uma fundamentação teórica sólida, o expressivo nível de acerto nas classificações obtido de forma recorrente pelos trabalhos desenvolvidos nas últimas décadas sugere que a insolvência empresarial é um evento que pode ser previsto com sucesso. Em conseqüência, os modelos de risco de crédito desenvolvidos empiricamente ainda possuem um forte apelo prático, sobretudo pela sua aplicabilidade nas atividades de concessão e gerenciamento de risco de crédito das instituições financeiras. 


\section{Referências}

ALTMAN, E. I: Financial ratios, discriminant analysis and the prediction of corporate bankruptcy. The Journal of Finance, v. 23, n. 4, p. 589-609, 1968.

ALTMAN, E. I. et al. Previsão de problemas financeiros em empresas, Revista de Administração de Empresas, v. 19, p. 17-28, 1979.

ANDRADE, F. W. M. Modelos de Risco de Crédito. Tecnologia de Crédito, n. 38, p. 23-53, 2003.

ASSAF NET, A. Estrutura e análise de balanços. 7. ed. São Paulo: Atlas, 2002.

BEAVER, W. H. Financial ratios as predictors of failure. Journal of Accounting Research, Empirical Research in Accounting: Selected Studies, v. 4, p. 77-111, 1966.

BESSIS, J. Risk management in banking. Chichester: John Wiley \& Sons, 1998.

CAOUETTE, J. B. et al. Gestão do risco de crédito: o próximo grande desafio financeiro. Rio de Janeiro: Qualitymark, 1999.

CASA NOVA, S. P. C.; ONUSIC, L. M. Mapeamento dos estudos sobre a utilização de Análise por Envoltória de Dados (DEA) na previsão de insolvência. Brasília: UnB Contábil, 2005. p. 207-238.

HAIR JR., J. F. et al. Multivariate data analysis. 5th ed. New Jersey: Prentice Hall, 1998.

HOSMER, D. W.; LEMESHOW, S. A goodness-of-fit test for the multiple logistic regression model. Communications in Statistics, A10, p.1043$1069,1980$.

KANITZ, S. C. Indicadores contábeis e financeiros de previsão de insolvência: a experiência na pequena e média empresa brasileira. São Paulo: 1976. Tese (Livre Docência) - Faculdade de Economia, Administração e Contabilidade, Universidade de São Paulo.

LACHENBRUCH, P. A. An almost unbiased method of obtaining confidence intervals for the probability of misclassification in discriminant analysis. Biometrics, v. 23, p. 639-645, 1967.

MATIAS, A. B. Contribuição às técnicas de análise financeira: um modelo de conceșsão de crédito. Trabalho apresentado ao Departamento de Administração da Faculdade de Economia, Administração e Contabilidade da Universidade de São Paulo. São Paulo: 1978.

NEOPHYTOU, E. et al. Predicting corporate failure: empirical evidence for the UK. University of Southampton, Working Paper, 2000.

OHLSON, J. A. Financial ratios and the probabilistic predictions of bankruptcy. Journal of Accounting Research, v. 18, n. 1, p. 109-131, 1980.

OLIVEIRA, J. G. C.; ANDRADE, F. W. M. Comparação entre medidas de performance de modelos de credit scoring. Tecnologia de Crédito, $\mathrm{n}$. 33, p. 35-47, 2002.

SANVICENTE, A. Z;; MINARDI, A. M. A. F. Identificação de indicadores contábeis significativos para a previsão de concordata de empresas. Instituto Brasileiro de Mercado de Capitais, Working Paper, 1998.

SILVA; J. P. Gestão e análise de risco de crédito. 4. ed. São Paulo: Atlas, 2003.

\section{NOTA - Endereço dos autores}

Universidade de São Paulo

Faculdade de Economia, Administração e Contabilidade

Pós-graduação em Ciências Contábeis

Av. Prof. Luciano Gualberto, 908 - prédio 3 - Cidade Universitária

São Paulo - SP

05508-900
Universidade de São Paulo

Faculdade de Economia, Administração e Contabilidade

Departamento de Contabilidade

Av. dos Bandeirantes, 3900 - Campus Universitário da USP

Ribeirão Preto - SP

14040-900 